\section{Construction of Recombi- nant Vaccinia Viruses Using PUV-Inactivated Virus as a Helper}

BioTechniques 31:534-540 (September 2001)

\section{ABSTRACT}

Recombinant vaccinia viruses $(V V s)$ are widely used as expression vectors in molecular biology and immunology and are now under evaluation for gene therapy. The current techniques for inserting foreign DNA into the large $V V$ genome are based on either homologous recombination between transfer plasmids and VV genomes or direct DNA ligation and packaging using replication-deficient poxviruses. Here, we describe efficient new versions of both methods that produce 90\%-100\% of the recombinant viruses. In the new homologous recombination method, VV DNA “arms" obtained by NotI digestion and intact transfer plasmids were used for co-transfection. In the direct DNA ligation method, foreign DNA was inserted into a unique NotI restriction site of the VV genome. In both methods, the generation of recombinant viruses was carried out in cells infected with a non-replicating, psoralen-UV (PUV)-inactivated helper $V V$. The convenience of these new techniques is demonstrated by the construction of recom binant VVs that produce E. coli $\beta$-galactosidase. An important feature of these strategies is that any VV strain can be used as a helper virus after PUV inactivation.

\section{INTRODUCTION}

Vaccinia virus (VV), a member of the Poxviridae, has a large, complex virus particle and a dsDNA genome of about $200 \mathrm{~kb}$. Several advantages of $\mathrm{VV}$ as a vector have made it a popular choice for gene delivery in vaccine studies (11) and therapeutic applications $(5,17)$. For example, VV has a large capacity for cloning foreign genes and infecting a broad range of animal and human cells.

The classical technique for inserting foreign DNA into the large VV genome is based on homologous recombination between transfer plasmids and VV genomes $(3,8,13)$. However, this method is labor intensive because of the low frequency of recombination and high background of parental viruses contam inating the virus plaques. Therefore, this method requires multiple cycles of purification of the recombinant viruses. Alternatively, direct DNA ligation can be used to construct recombinant VV using special helper poxvirus strains for the recovery of viable recombinant virus $(9,10,12,15)$. Although this method is effective and allows the cloning of inserts at least $26 \mathrm{~kb}$ in length (10), it has not been widely used because of the inconveniences associated with grow ing helper viruses and their low yield.

In this report, we demonstrate that VV inactivated with psoralen and longwave UV irradiation (PUV-VV) can be effectively used as a helper virus for the recovery of recombinant viruses generated either by direct DNA ligation or by homologous recombination between co-transfected transfer plasmids and viral DNA arms. Both new methods are equally efficient and allow the generation of recombinant $\mathrm{VV}$ with almost no background of parental viruses.

\section{MATERIALS AND METHODS}

\section{Cells and Viruses}

CV-1 cells (ATCC, Rockville, MD, USA) were grown in DMEM (Sigma, St. Louis, MO, USA) containing 5\% FBS and antibiotic-antimycotic solution (Sigma). The Lister (LIVP) strain of VV was used in these experiments as a parental virus. $\mathrm{VV}$ was propagated in $\mathrm{CV}-1$ cells and purified through a sucrose gradient as previously described (16).

\section{Plasmid Constructs}

For the construction of pNT8, the Not I region coding sequence of $\mathrm{VV}$ was amplified by PCR using forward primer 5'-GGGAATTCTTATACATCCTGTTCTATC-3', reverse primer 5'CCAAGCTTATGAGGAGTATTGCG GGGCTAC-3', and VV DNA as a template. The resulting 972-bp fragment contained flanking EcoRI and HindIII restriction sites at the $5^{\prime}$ and $3^{\prime}$ ends, respectively. The PCR product was cleaved with HindIII and EcoRI and was inserted into the pUC28 (1), resulting in pNT7. A 54-bp polylinker with multiple cloning sites was inserted into the NotI site of pNT7, generating pNT8, which was used as a vector for the cloning of foreign genes.

The BamHI-SmaI fragment (3293 bp) of pSC65 containing the $\mathrm{pE} / \mathrm{L}$ promoter was isolated by digestion with restriction enzymes, blunted with Klenow enzyme, and cloned into the SmaI site of the pNT8, resulting in pNZ2. The pNZ2 contains the cDNA of E. coli lacZ under the control of the VV early/late promoter $\mathrm{p} 7.5$ and a synthetic early/late VV $\mathrm{pE} / \mathrm{L}$ promoter derived from pSC65 plasmid (4). The pNZ2 was used for the construction of the following recombinant VVs: rVV-NotLZ1, rVV-Not-LZ2, and rVV-Not-PZ.

\section{DNA Ligation}

Purified VV DNA $(10 \mu \mathrm{g})$ was incubated overnight at $37^{\circ} \mathrm{C}$ in a $30-\mu \mathrm{L}$ reaction mixture containing $40 \mathrm{U}$ NotI. Digested DNA was purified by phenolchloroform extraction and treated with $2 \mathrm{U}$ alkaline phosphatase (Roche Molecular Biochemicals, Indianapolis, IN, USA) at $37^{\circ} \mathrm{C}$ for $50 \mathrm{~min}$. The reaction mixture was then heated at $65^{\circ} \mathrm{C}$ for 10 min, and the DNA was purified by phenol-chloroform extraction and dissolved in $15 \mu \mathrm{L}$ distilled water. The NotI-digested viral DNA $(1 \mu \mathrm{g})$ was ligated with $3 \mu \mathrm{g}$ of the 3357-bp NotI fragment of pNZ2, containing the p7.5-driven lac $Z$ gene, in a $20-\mu \mathrm{L}$ reaction mixture with 20 U T4 DNA ligase (New England Biolabs, Beverly, MA, USA) by being incubated overnight at room tem perature. The ligation mixture was used for the transfection experiment.

\section{Generation of PUV-VV}

$\mathrm{VV}\left(3 \times 10^{8} \mathrm{pfu} / \mathrm{mL}\right)$ was incubated in a $35-\mathrm{mm}$ plate with $1 \mu \mathrm{g} / \mathrm{mL}$ psoralen (4'-aminomethyl-trioxsalen; Calbiochem-Novabiochem, San Diego, CA, USA) in a total volume of $1 \mathrm{~mL}$ Hanks buffer containing $0.1 \%$ BSA at room temperature for $10 \mathrm{~min}$. The plate was irradiated for 6-8 min in a Stratalinker ${ }^{\circledR} 1800$ UV Crosslinker (Stratagene, La Jolla, CA, USA) equipped with five 365 -nm, long-wave UV bulbs as previously described (17). The PUV- 


\section{Short Technical Reports}

VV was assayed for remaining viable virus particles by standard plaque assay (3). The PUV-VV that did not induce cytopathic effects on CV-1 cells within three days after infection was used for the experiments.

\section{Generation of Recombinant VVs}

$\mathrm{CV}-1$ cells grown in $60-\mathrm{mm}$ dishes were infected with PUV-VV at an MOI of one. Two hours after infection, the cells were transfected with either the previously described ligation mixture or with $2 \mu \mathrm{g}$ of the mixture of NotI-digested viral DNA and $6 \mu \mathrm{g}$ intact plasmid DNA. The lipid-mediated transfection of cells was carried out using $5 \mu \mathrm{L}$ GenePORTER $^{\mathrm{TM}}$ reagent (Gene Therapy Systems, San Diego, CA, USA) per
$1 \mu \mathrm{g}$ DNA, according to manufacturer's instructions. The cells were incubated in the transfection mixture for $4 \mathrm{~h}$ and then supplemented with a medium containing 20\% FBS. Cytopathic effects were monitored daily by light microscopy. The cells were incubated for 5-8 days, until virus plaques formed and the cytopathic effect was complete. The infected cells were then harvested, resuspended in $0.5 \mathrm{~mL}$ medium, and frozen and thawed three times to release the virus. Single virus plaques were selected for the preparation of small and large recombinant virus stocks and analyzed for the lac Z gene insertion and expression using Southern blot (17) and plaque assay (3) involving blue color production after using the substrate for $\beta$-galactosidase X-Gal (Molecular Probes, Eugene, OR, USA), respectively.

\section{RESULTS AND DISCUSSION}

We described two alternative new protocols for the construction of recombinant VVs. The first method is based on the ligation of a foreign DNA fragment with the VV DNA arms produced by NotI digestion, followed by transfection of CV-1 cells with this ligation mixture. The second method is based on homologous recombination in transfected cells between the VV DNA arms produced by Not I digestion and intact transfer plasmids containing a foreign gene expression cassette flanked with VV sequences. In both methods, before transfection, cells were infected with a PUV-VV, which served as a helper for the initiation of the replication cycle for the recombinant virus. The unique Not I site in VV is located in the HindIII F region and is nonessential for virus replication (6). The presence of the unique NotI restriction site in the VV genome of strain Lister was confirmed by pulsed field electrophoresis of NotI-digested viral DNA (data not shown).

Plasmid pNZ2 was used to test both protocols for the efficient generation of recombinant VV (Figure 1). The first method is based entirely on the ligation of DNA fragments and generation of recombinant genomes in vitro, followed by the transfection of CV-1 cells 
previously infected with PUV-VV (Figure 1A). Genomic DNA of recombinant viruses (designated rVV-Not-LZ1 and rVV-Not-LZ2) contained inserts in two alternative orientations as shown in Figure 1A. The second method, for which DNA ligation is not required, is based on intracellular recombination between homologous VV sequences within the intact plasmid (a derivative of $\mathrm{pNZ2}$ ) and viral DNA cleaved at a unique NotI site (Figure 1B). Recombinant virus designated rVV-Not-PZ was obtained using this method.

By both methods, the expression cassette of foreign DNA was inserted precisely at the NotI site as predicted. The integration of the lac $Z$ gene into the VV genome was confirmed by Southern blot hybridization (Figure 2, panels $A$ and $B$ ). The purity of recom binant VV plaques was analyzed by plaque assay. Using our transfection-recombination method, approximately $90 \%$ of the virus plaques acquired blue color, while about $10 \%$ of the plaques remained color-free (Figure 2, panels C

Table 1. Efficiency of Foreign Gene Insertion into VV Genome

\begin{tabular}{|c|c|c|c|c|c|c|c|}
\hline \multirow{3}{*}{$\begin{array}{l}\text { Method of } \\
\text { Transfection }\end{array}$} & \multicolumn{6}{|c|}{$\begin{array}{l}\text { No. of Recombinant (R) and Wild-Type (wt) } \\
\text { Virus Plaques }\end{array}$} & \multirow{3}{*}{$\begin{array}{c}\text { Recombinant } \\
\text { Viruses (\%) } \\
\text { mean } \pm \text { SD }\end{array}$} \\
\hline & \multicolumn{2}{|c|}{ Exp. 1} & \multicolumn{2}{|c|}{ Exp. 2} & \multicolumn{2}{|c|}{ Exp. 3} & \\
\hline & $\mathrm{R}$ & wt & $\mathrm{R}$ & wt & $\mathrm{R}$ & wt & \\
\hline$A$ & 90 & 0 & 54 & 1 & 85 & 0 & $99.3 \pm 1.15$ \\
\hline$B$ & 158 & 6 & 80 & 20 & 140 & 0 & $92.2 \pm 10.74$ \\
\hline $\mathrm{C}$ & 24 & $>500$ & 15 & $>500$ & 32 & $>500$ & $<0.18$ \\
\hline
\end{tabular}

and D). To confirm the purity of the obtained recombinant clones, individual blue plaques were isolated, and viruses were propagated and then titered again in the agar medium with X-Gal. All of the generated virus plaques were blue, indicating that they contained pure, homogeneous recombinant $\mathrm{VV}$.

Both strategies produced an almost background-free yield of recombinant viruses. The efficiency of recombinant virus production was evaluated and compared by plaque assay in three methods using the same transfer plasmid pNZ2 (Table 1). In addition to the direct ligation-transfection (A) and cotransfection-recombination (B) protocols, the traditional homologous recom bination method (C) was also performed. The traditional method involved cell infection with a live VV, followed by transfection with the transfer
A

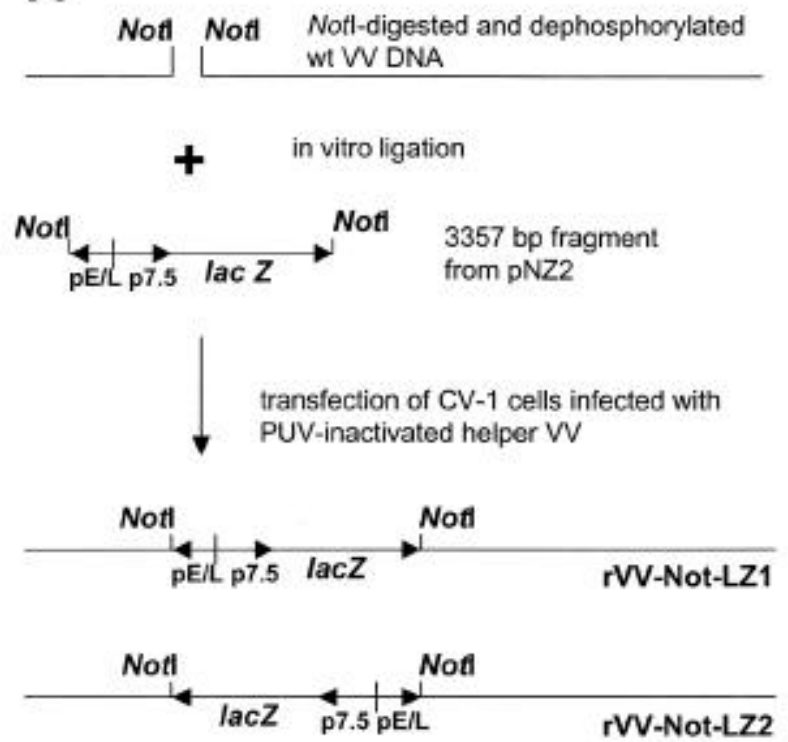

B
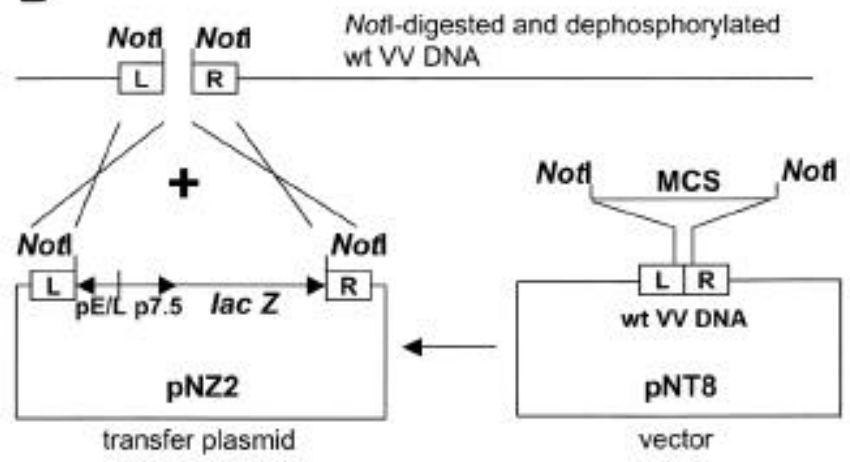

transfection of CV-1 cells infected with PUV-inactivated helper W: homologous recombination

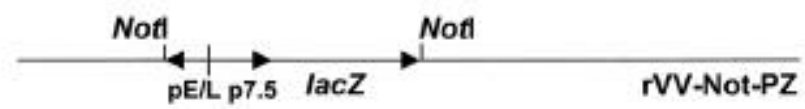

Figure 1. Strategies for recombinant VV production. (A) DNA ligation method. Wild-type VV (strain Lister) DNA digested at the unique NotI restriction site (DNA arms) was treated with alkaline phosphatase and ligated with the NotI fragment from the pNZ2 plasmid. The ligation mixture was transfected into PUVVV-infected CV-1 cells for recombinant VV generation. Recombinants rVV-Not-LZ1 and rVV-Not-LZ2 with the lacZ gene expression cassette in opposite orientation were obtained. (B) New homologous recombination method. PUV-VV-infected cells were transfected with VV DNA arms and intact pNZ2 to produce the rVV-Not-PZ. L and R cubes show VV sequences involved in homologous recombination. Although the sequence analysis has not been performed, we anticipate that the DNA structures of resultant rVV-Not-LZ1 and rVV-Not-PZ recombinant viruses are also identical. Vector pNT8 was used for the construction of plasmid DNA pNZ2. 


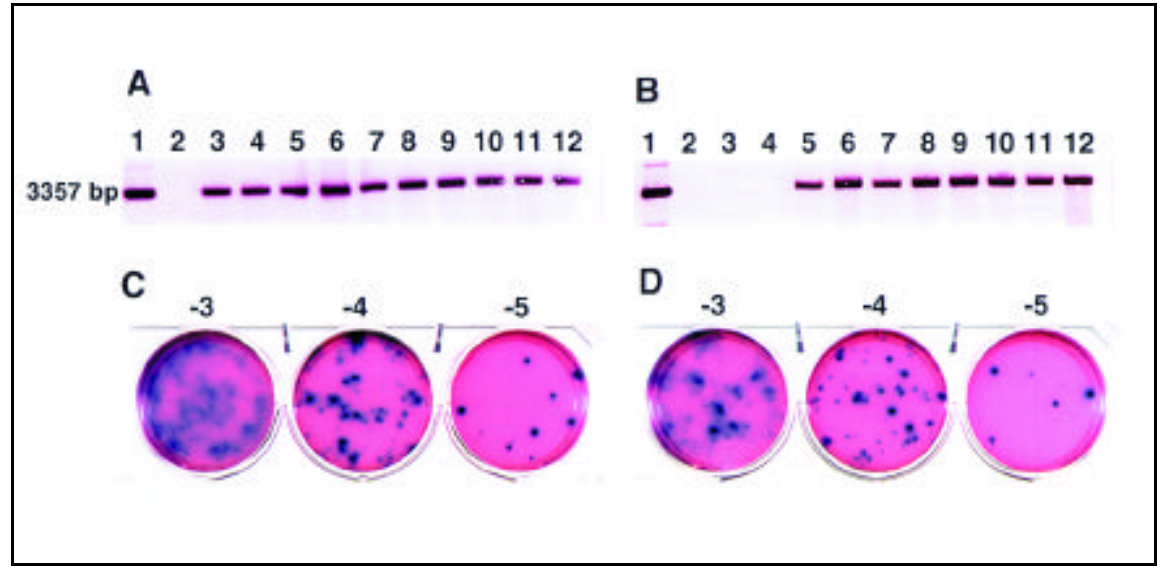

Figure 2. Gene insertion and expression analyses. (A and B) Southern blot analysis. Recombinant VV DNAs were digested with NotI, electrophoresed on a $0.8 \%$ agarose gel, and analyzed using a 3357-bp DNA probe of pNZ2. Data show that recombinant viruses produced by the DNA ligation method (A) or by the new homologous recombination method (B) contain lacZ gene inserted at the NotI site. Lane 1, positive control, NotI fragment (3357 bp) of plasmid pNZ2; lane 2, DNA from CV-1 cells infected with wild-type VV; (A) lanes 3-12 and (B) lanes 5-12, DNAs from the cells infected with viruses from single blue plaques; (B) lanes 3 and 4, DNAs from the cells infected with viruses from two white plaques. Recombinant virus plaques obtained using the DNA ligation method $(\mathrm{C})$ or the new homologous recombination method (D). CV-1 cells grown on six-well plates were infected with 10 -fold dilutions $\left(10^{-3}, 10^{-4}\right.$, and $10^{-5}$ ) of viruses obtained on days 5-8 after the transfection. After $48 \mathrm{~h}$, the cells were overlaid with agar medium containing the $\mathrm{X}-\mathrm{Gal}$ to visualize the recombinant blue plaques. plasmid pNZ2. Almost 100\% (99.3\%) of the virus plaques produced by the DNA ligation method were recombinant, and $92.2 \%$ of the recombinant virus plaques were obtained by the cotransfection-recombination method. Only $0.18 \%$ of the virus plaques were recombinant using the traditional method. An earlier study demonstrated that lacZ gene-based screening VV recom binants represented about $0.1 \%$ of the total viral progeny (3). Thus, both new strategies for the construction of recom binant VV were highly efficient in the generation of recombinants. This is due to the use of $\mathrm{VV}$ arms for transfection, which can be rescued either by ligation with the foreign DNA or by the new homologous recombination method using the transfer plasmid. Previously, when an insertion was directed into the thymidine kinase (TK) locus of the VV genome, usually between $10 \%$ and $80 \%$ of TK plaques were recombinant $(3,8)$. 
The main advantage of these alternative methods is that any poxvirus strain, including VV itself, can be inactivated by PUV and used as a helper virus for the production of recombinant viruses. The properties of non-replicating PUV-VV have recently been reported $(7,14,18$; unpublished data). Once inactivated, a virus can provide the early functions required for replication and maturation of transfected viral DNA but cannot replicate and mature into infectious particles. The high efficacy of recombinant virus production and the purity of the plaques were achieved by complete digestion of VV genomic DNA at a unique Not I site and by the use of an inactivated helper virus. Therefore, it does not require reporter genes and multiple plaque purification cycles, which are needed for most methods of recombinant VV constructions. Furthermore, for the first time, we demonstrated that intracellular homologous recombination between DNA arms obtained at a unique restriction site and intact naked plasmids in the presence of a helper virus could rescue the genomic viral DNA to produce recombinant $\mathrm{VV}$. We have recently used this strategy to generate herpesviruses as well (2). The DNA ligation method could be more convenient for the generation of recombinant viruses that express toxic gene products or when foreign DNA is inserted into a newly created unique restriction site of the VV genome. When a series of recombinant viruses carrying foreign DNA at the same locus are required, the homologous recombination method could be a better choice. An important requirement of these methods is the presence of a unique restriction site on the VV genome. Here, we took advantage of the unique NotI site present in most VV strains. However, other unique restriction sites can be created in the VV genome by genetic manipulation (9) and used for insertion of a foreign gene expression cassette.

In summary, we report two new methods for the fast and efficient construction of recombinant VV. Neither virus purification cycles nor reporter gene selection is necessary because $90 \%-100 \%$ of the VV progeny carry the foreign DNA. An important feature of these strategies is that any VV strain can be used as a helper virus after PUV inactivation. These methods should help to advance the development of VV vector systems for vaccine and gene therapy studies. They can be also used in functional studies to investigate the virus replication cycle and packaging if various early mutants of $\mathrm{VV}$ are used as the helpers.

\section{REFERENCES}

1.Benes, V., Z. Hostomsky, L. Arnold, and V. Paces. 1993. M13 and pUC vectors with new unique restriction sites for cloning. Gene 130:151-152.

2.Boldogkoi, Z., A. Braun, J. Antal, and I. Fodor. 1998. A restriction cleavage and transfection system for introducing foreign DNA into the unique and inverted repeat regions of a herpesvirus. Res. Virol. 149:87-97.

3.Chakrabarti, S., K. Brechling, and B. Moss. 1985. Vaccinia virus expression vector: coexpression of $\beta$-galactosidase provides visual screening of recombinant virus plaques. Mol. Cell. Biol. 5:3403-3409.

4.Chakrabarti, S., J.R. Sisler, and B. Moss. 1997. Compact, synthetic, vaccinia virus early/late promoter for protein expression. BioTechniques 23:1094-1097.

5.Chen, B., T.M. Timiryasova, M.L. Andres, E.H. Kajioka, R.D. Roy, D.S. Gridley, and I. Fodor. 2000. Evaluation of combined vaccinia virus-mediated antitumor gene therapy with p53, IL-2 and IL-12 in a glioma model. Cancer Gene Ther. 7:1437-1447.

6.Goebel, S.J., G.P. Johnson, M.E. Perkus, S.W. Davis, J.P. Winslow, and E. Paoletti. 1990. The complete DNA sequence of vaccinia virus. Virology 179:247-266.

7.Meko, J.B., K. Tsung, and J.A. Norton. 1996. Cytokine production and antitumor effect of a nonreplicating, noncytopathic recom binant vaccinia virus expressing interleukin12. Surgery 120:274-281.

8.Mackett, M., G.L. Smith, and B. Moss. 1982. Vaccinia virus: a selectable eukaryotic cloning and expression vector. Proc. Natl. Acad. Sci. USA 79:7415-7419.

9.Merchlinsky, M., D. Eckert, E. Smith, and M. Zauderer. 1997. Construction and characterization of vaccinia direct ligation vectors. Virology 238:444-451.

10.Merchlinsky, M. and B. Moss. 1992. Introduction of foreign DNA into the vaccinia virus genome by in vitro ligation: recombination-independent selectable cloning vectors. Virology 190:522-526.

11.Moss, B. 1996. Genetically engineered poxviruses for recombinant gene expression, vaccination, and safety. Proc. Natl. Acad. Sci. USA 93:11341-11348.

12.Pfleiderer, M., F.G. Falkner, and F. Dorner. 1995. A novel vaccinia virus expression system allowing construction of recombinants without the need for selection markers, plasmids and bacterial hosts. J. Gen. Virol. 76:2957-2962.

13.Piccini, A., M.E. Perkus, and E. Paoletti. 1987. Vaccinia virus as an expression vector. Methods in Enzymol. 153:545-563.

14.Puhlmann, M., C.K. Brown, M. Gnant, J. Huang, S.K. Libutti, H.R. Alexander, and D.L. Bartlett. 2000. Vaccinia as a vector for tumor-directed gene therapy: biodistribution of a thymidine kinase-deleted mutant. Cancer Gene Ther. 7:66-73.

15.Scheiflinger, F., F. Dorner, and F.G. Falkner. 1992. Construction of chimeric vaccinia viruses by molecular cloning and packaging. Proc. Natl. Acad. Sci. USA 89:9977-9981.

16.Timiryasova, T.M., T.N. Kopylova-Sviridova, and I. Fodor. 1993. Analysis of expression of the reporter genes in different regions of vaccinia virus genome. Mol. Biol. 27:392401.

17.Timiryasova, T.M., J. Li, B. Chen, D. Chong, W.H.R. Langridge, D.S. Gridley, and I. Fodor. 1999. Antitumor effect of vaccinia virus in glioma model. Oncol. Res. $11: 133-144$

18.Tsung, K., J.H. Yim, W. Marti, R.M.L. Buller, and J.A. Norton. 1996. Gene expression and cytopathic effect of vaccinia virus inactivated by psoralen and long-wave UV light. J. Virol. 70:165-171.

We gratefully acknowledge Dr. B. Moss (National Institute of Allergy and Infectious Diseases, NIH, Bethesda, MD, USA) for pSC65 plasmid. We also thank Dr. A.J. Zuccarelli for advice, G. Harding for expert technical assistance in the pulsed field electrophoresis experiment, and Dr. S. Hilliker for critical reading of the manuscript. Address correspondence to Dr. Istvan Fodor, Center for Molecular Biology \& Gene Therapy, School of Medicine, Loma Linda University, 11085 Campus Street, Mortensen Hall, Loma Linda, CA 92350, USA. e-mail: ifodor@som.llu.edu

Received 17 November 2000; accepted 16 March 2001.

\section{Tatyana M. Timiryasova, Bing Chen, Nadja Fodor, and Istvan Fodor School of Medicine Loma Linda University Loma Linda, CA, USA}

For reprints of this or any other article, contact Reprints@BioTechniques.com 